\title{
LAPLACE TRANSFORM IDENTITIES FOR DIFFUSIONS, WITH APPLICATIONS TO REBATES AND BARRIER OPTIONS
}

\author{
HARDY HULLEY \\ School of Finance and Economics \\ University of Technology, Sydney \\ P.O. Box 123, Broadway, NSW 2007, Australia \\ E-mail: hardy.hulley@uts.edu.au \\ ECKHARD PLATEN \\ School of Finance and Economics and Department of Mathematical Sciences \\ University of Technology, Sydney \\ P.O. Box 123, Broadway, NSW 2007, Australia \\ E-mail: eckhard.platen@uts.edu.au
}

\begin{abstract}
We start with a general time-homogeneous scalar diffusion whose state space is an interval $I \subseteq \mathbb{R}$. If it is started at $x \in I$, then we consider the problem of imposing upper and/or lower boundary conditions at two points $a, b \in I$, where $a<x<b$. Using a simple integral identity, we derive general expressions for the Laplace transform of the transition density of the process, if killing or reflecting boundaries are specified. We also obtain a number of useful expressions for the Laplace transforms of some functions of first-passage times for the diffusion. These results are applied to the special case of squared Bessel processes with killing or reflecting boundaries. In particular, we demonstrate how the above-mentioned integral identity enables us to derive the transition density of a squared Bessel process killed at the origin, without the need to invert a Laplace transform. Finally, as an application, we consider the problem of pricing barrier options on an index described by the minimal market model.
\end{abstract}

1. Introduction. The theory of time-homogeneous linear scalar diffusions is elegant and classical, with Borodin and Salminen [4], Itô and McKean [16] and Karlin and Taylor [17]

2000 Mathematics Subject Classification: Primary 60J60, 91B28; Secondary 44A10, 47D07, $60 \mathrm{~J} 70$.

Key words and phrases: diffusions, transition densities, first-passage times, Laplace transforms, squared Bessel processes, minimal market model, real-world pricing, rebates, barrier options.

The paper is in final form and no version of it will be published elsewhere. 
featuring as prominent references. An important object in this theory is the infinitesimal operator of such a process. The Laplace transform of the transition density of a diffusion, as well as the Laplace transforms of its first-passage time densities, may be expressed in terms of the fundamental solutions of the eigenvalue problem for this operator.

Section 2 of our paper contains a brief overview of the above theory, in which we rely heavily on the excellent account of Borodin and Salminen [4, Chap. II], especially for style and notation. We do, however, add one new ingredient to the presentation. This is an integral equation of convolution type, described by Peskir [23] as a "ChapmanKolmogorov equation of Volterra type", that relates the transition density of a diffusion with its first-passage time densities. By combining this relation with the above-mentioned representations of the Laplace transforms of the transition density and first-passage time densities, we are able to derive a number of useful identities by purely formal algebraic manipulation. For example, the transition densities of diffusions constructed from an original process by killing or reflecting it at interior points of its state space are readily obtained in this way - at least, up to Laplace transform. We also present some ancillary identities for the Laplace transforms of expressions involving first-passage times. A few of these originate from Davidov and Linetsky [7], but the arguments used there are different. However, two of the identities are new, and are used later to obtain expressions for rebate prices.

Section 3 applies the above theory to an important class of diffusions that play a central role in this paper, namely squared Bessel processes. Although we mainly summarize results from Borodin and Salminen [4, App. 1.23], Göing-Jaeschke and Yor [14] and Revuz and Yor [25, Chap. XI], the section does contain a novel derivation of the transition density of a squared Bessel process of dimension less than two, killed at the origin. This function is apparently not very well known, which is surprising, since it is manifestly useful. For example, in our opinion, it yields the most direct derivations of European option prices for the constant elasticity of variance (CEV) model of Cox [5]. One of the few places where the transition density in question does appear is Borodin and Salminen [4, p. 136], where it was obtained by inverting the appropriate Green's function. Our derivation, on the other hand, requires no inversion of a Laplace transform. Instead, we start with the previously mentioned integral equation, and proceed directly.

Section 4 is devoted to a brief exposition of the minimal market model (MMM). This is the workhorse of the so-called benchmark approach to contingent claim valuation, and was originally developed as a concrete model for the growth optimal portfolio (GOP) of Kelly [18]. A salient feature of the GOP, originally observed by Long [21], is that all selffinancing portfolios are local martingales under the real-world probability measure, when denominated in units of this portfolio. This facilitates a "real-world martingale pricing theory", in which the price of a claim is determined by computing the expected value of its numéraire-denominated payoff under the real-world measure, with the GOP chosen as numéraire. An obvious advantage of this is that it obviates the need for heavy changeof-measure machinery, such as Girsanov's theorem. This is not insignificant, because although the existence of an equivalent risk-neutral probability measure is often invoked casually, the justification for such a step is sometimes quite subtle - as demonstrated 
in Delbaen and Shirakawa [9] and Heath and Platen [15] — and hinges on the delicate question of whether the putative density process is a proper martingale, or merely a local martingale. A further advantage of the benchmark approach is enhanced modelling flexibility. This arises because the existence of a GOP is necessary, but not sufficient, for the existence of an equivalent risk-neutral probability measure. In particular, there are models for which real-world pricing is consistent, even though they do not admit any equivalent risk-neutral probability measures; in fact, the MMM is an example of such. For a detailed account of the MMM, and the benchmark approach in general, the reader is directed to Platen and Heath [24].

A crucial feature of the MMM is that it admits a representation as a scaled and deterministically time-changed squared Bessel process of dimension four. Since the transition densities and various other analytic properties of squared Bessel processes are known from Section 3, we are able to obtain convenient pricing formulae for contingent claims written on a large equity index with MMM dynamics. In particular, Sections 5 and 6 examine, respectively, the pricing of rebates and barrier options whose payoffs are determined by whether or not the index breaches an exponential barrier before expiry. The barrier we specify is in fact proportional to the scaling factor in the above-mentioned representation, implying that the payoffs of the claims in question are dependent upon whether or not a squared Bessel process of dimension four hits a constant level within a certain period. The results from Section 2 are thus applicable, allowing us to derive expressions for the Laplace transforms of the prices of these instruments. Actual prices are then obtained by numerical inversion of the transformed prices.

While the Laplace transform is perhaps not the most popular tool for analyzing exotic financial derivatives, there are a number of instances of its use. Probably the first and most widely cited of these is Geman and Yor [12], where an expression was obtained for the Laplace transform of the price of an Asian option on a security following a geometric Brownian motion. The numerical aspects of inverting this particular expression have been the focus of a number of studies, including Craddock et al. [6], Fu et al. [10], Geman and Eydeland [11] and Shaw [26, Chap. 10]. Within the same framework as above, Davidov and Linetsky [8] also considered double-barrier step options. Once again, option pricing formulae were derived, up to Laplace transform, and then inverted numerically. Double barrier options on a security following a geometric Brownian motion were the subject of Pelsser [22]. The approach there employed contour integration to perform analytic inversion, yielding series expansions for the prices of the instruments considered. Finally, Davidov and Linetsky [7] priced rebates, lookback options and barrier options on a security with CEV dynamics, by numerical Laplace transform inversion. We acknowledge the influence of the latter article, in particular, on our work.

The majority of the studies cited above rely on the Euler method, presented in Abate and Whitt [1], for numerical inversion of Laplace transforms; as do we. The attraction of this scheme is that it is relatively quick, without appearing to compromise on accuracy. Nevertheless, one cannot be sure, since there are no guaranteed error bounds - a drawback of all inversion algorithms. Furthermore, Craddock et al. [6] have reported that inversion schemes, in general, appear quite sensitive to model parameters. This apparent lack of 
robustness, together with the computational effort required and the absence of error bounds, make us hesitant to endorse numerical Laplace transform inversion unreservedly as a practical technique for valuing exotic options. Ultimately, one must bear in mind that the computation of an unbounded linear operator, such as the inverse Laplace transform, is inherently unstable. Nevertheless, the approach undoubtedly has a role to play, even if only to compare its results with those of other methods. Furthermore, modern computer hardware has made the execution times of inversion algorithms a feasible proposition.

2. Laplace transform identities for diffusions. Let $X=\left(X_{t}\right)_{t \geq 0}$ be a regular onedimensional time-homogeneous diffusion process, whose state space is an interval $I \subseteq \mathbb{R}$, which is typically $\mathbb{R},[0, \infty)$ or $(0, \infty)$. The local behaviour of $X$ is expressed by its infinitesimal generator, which we take to be a second-order linear differential operator $\mathcal{G}: \operatorname{Dom}(\mathcal{G}) \rightarrow \mathrm{C}_{\mathrm{b}}(I)$, given by

$$
\mathcal{G} f(x):=\frac{1}{2} \sigma^{2}(x) f^{\prime \prime}(x)+\mu(x) f^{\prime}(x),
$$

for all $f \in \operatorname{Dom}(\mathcal{G})$ and $x \in \stackrel{\circ}{I}$, where $\stackrel{\circ}{I}$ denotes the interior of $I$ and $\mathrm{C}_{\mathrm{b}}(I)$ is the space of all bounded continuous functions on $I$. In the expression above, $\sigma(\cdot)$ is the diffusion coefficient of the process, while $\mu(\cdot)$ is its drift coefficient. We assume that these functions are continuous on $I$, and that $\sigma(x)>0$, for all $x \in I$. The reader is directed to Borodin and Salminen [4, p. 16] for a detailed description of the operator's domain $\operatorname{Dom}(\mathcal{G}) \subseteq \mathrm{C}_{\mathrm{b}}(I)$.

The basic characteristics of $X$ are its speed density $\mathfrak{m}(\cdot)$ and scale function $\mathfrak{s}(\cdot)$. These may be expressed in terms of the drift and diffusion coefficients as follows:

$$
\mathfrak{s}(x):=\int^{x} \mathfrak{s}^{\prime}(\xi) d \xi \quad \text { and } \quad \mathfrak{m}(x):=\frac{2}{\sigma^{2}(x) \mathfrak{s}^{\prime}(x)},
$$

where the scale density is given by

$$
\mathfrak{s}^{\prime}(x):=\exp \left(-\int^{x} \frac{2 \mu(\xi)}{\sigma^{2}(\xi)} d \xi\right)
$$

for all $x \in I$.

We shall denote the transition density of $X$ with respect to its speed measure by $q(\cdot, \cdot, \cdot)$, so that

$$
\mathbb{P}_{x}\left[X_{t} \in A\right]=\int_{A} q(t, x, y) \mathfrak{m}(y) d y,
$$

for all $t \geq 0$ and $x \in I$, and for every Borel set $A \in \mathscr{B}(I)$. In this expression $\mathbb{P}_{x}$ denotes the probability measure under which $X$ starts at $x$ at time zero.

If we fix $\alpha>0$, then we may introduce the Green's function $G_{\alpha}(\cdot, \cdot)$ as the Laplace transform, with respect to time, of the transition density of $X$ :

$$
G_{\alpha}(x, y):=\mathscr{L}_{\alpha}\{q(t, x, y)\}=\int_{0}^{\infty} e^{-\alpha t} q(t, x, y) d t,
$$

for all $x, y \in I$. The Green's function may be factorized as follows:

$$
G_{\alpha}(x, y)= \begin{cases}w_{\alpha}^{-1} \psi_{\alpha}(x) \phi_{\alpha}(y) & \text { if } x \leq y \\ w_{\alpha}^{-1} \psi_{\alpha}(y) \phi_{\alpha}(x) & \text { if } x \geq y\end{cases}
$$


Here $\psi_{\alpha}(\cdot)$ and $\phi_{\alpha}(\cdot)$ are, respectively, the unique (up to a multiplicative constant) increasing and decreasing solutions to the equation

$$
\mathcal{G} u(x)=\alpha u(x),
$$

for all $x \in \stackrel{\circ}{I}$, subject to appropriate boundary conditions at the endpoints of $I$. Furthermore, the Wronskian

$$
w_{\alpha}:=\frac{\psi_{\alpha}^{\prime}(x) \phi_{\alpha}(x)-\psi_{\alpha}(x) \phi_{\alpha}^{\prime}(x)}{\mathfrak{s}^{\prime}(x)}
$$

is independent of $x \in \stackrel{\circ}{I}$.

For any $z \in I$, let

$$
\tau_{z}:=\inf \left\{t>0: X_{t}=z\right\}
$$

be the first-passage time of $X$ to $z$. We shall denote its density with respect to Lebesgue measure by $p_{z}(\cdot, \cdot)$, so that

$$
\mathbb{P}_{x}\left[\tau_{z} \leq t\right]=\int_{0}^{t} p_{z}(x, s) d s .
$$

Suppose now that $\tilde{q}_{z}(\cdot, \cdot, \cdot)$ is the transition density, with respect to speed measure, of $X$ killed at $z$, so that

$$
\mathbb{P}_{x}\left[X_{t} \in A, \tau_{z}>t\right]=\int_{A} \tilde{q}_{z}(t, x, y) \mathfrak{m}(y) d y,
$$

for all $A \in \mathscr{B}(I)$. Then the following fundamental relation underlies many of the derivations in this paper:

Lemma 1. Let $x, y, z \in I$ and suppose that $t>0$. Then

$$
q(t, x, y)=\tilde{q}_{z}(t, x, y)+\int_{0}^{t} p_{z}(x, s) q(t-s, z, y) d s .
$$

Proof. It follows from the Markov property of $X$ that

$$
\begin{aligned}
\mathbb{P}_{x}\left[X_{t} \leq y\right] & =\mathbb{P}_{x}\left[X_{t} \leq y, \tau_{z}>t\right]+\mathbb{P}_{x}\left[X_{t} \leq y, \tau_{z} \leq t\right] \\
& =\mathbb{P}_{x}\left[X_{t} \leq y, \tau_{z}>t\right]+\int_{0}^{t} \mathbb{P}_{x}\left[\tau_{z} \in d s\right] \mathbb{P}_{x}\left[X_{t} \leq y \mid \tau_{z}=s\right] \\
& =\mathbb{P}_{x}\left[X_{t} \leq y, \tau_{z}>t\right]+\int_{0}^{t} \mathbb{P}_{x}\left[\tau_{z} \in d s\right] \mathbb{P}_{z}\left[X_{t-s} \leq y \mid \tau_{z}=s\right] .
\end{aligned}
$$

Now differentiate with respect to $y$ and divide through by $\mathfrak{m}(y)$.

Note that if $t>0$ and $x \leq z \leq y$ or $x \geq z \geq y$, then $\tilde{q}_{z}(t, x, y)=0$. In that case the convolution property of Laplace transforms gives

$$
\mathscr{L}_{\alpha}\{q(t, x, y)\}=\mathscr{L}_{\alpha}\left\{p_{z}(x, t)\right\} \mathscr{L}_{\alpha}\{q(t, z, y)\},
$$

from which it follows that

$$
\mathbb{E}_{x}\left[e^{-\alpha \tau_{z}}\right]=\mathscr{L}_{\alpha}\left\{p_{z}(x, t)\right\}=\frac{G_{\alpha}(x, y)}{G_{\alpha}(z, y)}= \begin{cases}\frac{\psi_{\alpha}(x)}{\psi_{\alpha}(z)} & \text { if } x \leq z \\ \frac{\phi_{\alpha}(x)}{\phi_{\alpha}(z)} & \text { if } x \geq z\end{cases}
$$


This well-known formula is derived using a different argument in Itô and McKean [16, p. 128]. Using standard identities for Laplace transforms, we obtain the following useful expressions from (4):

Proposition 2. Fix $\alpha>0$ and let $t \geq 0$ and $x, z \in I$. Then

$$
\mathscr{L}_{\alpha}\left\{\mathbb{P}_{x}\left[\tau_{z} \leq t\right]\right\}= \begin{cases}\frac{1}{\alpha} \frac{\psi_{\alpha}(x)}{\psi_{\alpha}(z)} & \text { if } x \leq z \\ \frac{1}{\alpha} \frac{\phi_{\alpha}(x)}{\phi_{\alpha}(z)} & \text { if } x \geq z\end{cases}
$$

and

$$
\mathscr{L}_{\alpha}\left\{\mathbb{E}_{x}\left[\mathbb{I}_{\left\{\tau_{z} \leq t\right\}} e^{-\beta \tau_{z}}\right]\right\}= \begin{cases}\frac{1}{\alpha} \frac{\psi_{\alpha+\beta}(x)}{\psi_{\alpha+\beta}(z)} & \text { if } x \leq z ; \\ \frac{1}{\alpha} \frac{\phi_{\alpha+\beta}(x)}{\phi_{\alpha+\beta}(z)} & \text { if } x \geq z,\end{cases}
$$

for all $\beta>0$. Furthermore,

$$
\mathbb{E}_{x}\left[\left(\gamma+\lambda \tau_{z}\right)^{-\rho}\right]= \begin{cases}\frac{1}{\Gamma(\rho)} \mathscr{L}_{\gamma}\left\{s^{\rho-1} \frac{\psi_{\lambda s}(x)}{\psi_{\lambda s}(z)}\right\} & \text { if } x \leq z \\ \frac{1}{\Gamma(\rho)} \mathscr{L}_{\gamma}\left\{s^{\rho-1} \frac{\phi_{\lambda s}(x)}{\phi_{\lambda s}(z)}\right\} & \text { if } x \geq z\end{cases}
$$

and

$$
\mathscr{L}_{\alpha}\left\{\mathbb{E}_{x}\left[\mathbb{I}_{\left\{\tau_{z} \leq t\right\}}\left(\gamma+\lambda \tau_{z}\right)^{-\rho}\right]\right\}= \begin{cases}\frac{1}{\alpha \Gamma(\rho)} \mathscr{L}_{\gamma}\left\{s^{\rho-1} \frac{\psi_{\alpha+\lambda s}(x)}{\psi_{\alpha+\lambda s}(z)}\right\} & \text { if } x \leq z ; \\ \frac{1}{\alpha \Gamma(\rho)} \mathscr{L}_{\gamma}\left\{s^{\rho-1} \frac{\phi_{\alpha+\lambda s}(x)}{\phi_{\alpha+\lambda s}(z)}\right\} & \text { if } x \geq z\end{cases}
$$

for all $\gamma, \lambda, \rho>0$.

In order to interpret the above expressions, note that the Laplace transforms on the left-hand sides of (5), (6) and (8) are of the form $\mathscr{L}_{\alpha}\{f(t)\}=\hat{f}(\alpha)$, while the Laplace transforms on the right-hand sides of (7) and (8) are of the form $\mathscr{L}_{\gamma}\{f(s)\}=\hat{f}(\gamma)$.

Proof. Equation (5) follows from

$$
\mathscr{L}_{\alpha}\left\{\mathbb{P}_{x}\left[\tau_{z} \leq t\right]\right\}=\mathscr{L}_{\alpha}\left\{\int_{0}^{t} p_{z}(x, s) d s\right\}=\frac{1}{\alpha} \mathscr{L}_{\alpha}\left\{p_{z}(x, t)\right\} .
$$

To verify (6), note that

$$
\begin{aligned}
& \mathscr{L}_{\alpha}\left\{\mathbb{E}_{x}\left[\mathbb{I}_{\left\{\tau_{z} \leq t\right\}} e^{-\beta \tau_{z}}\right]\right\}=\mathscr{L}_{\alpha}\left\{\int_{0}^{t} e^{-\beta s} p_{z}(x, s) d s\right\}=\frac{1}{\alpha} \mathscr{L}_{\alpha}\left\{e^{-\beta t} p_{z}(x, t)\right\} \\
& =\frac{1}{\alpha} \mathscr{L}_{\alpha+\beta}\left\{p_{z}(x, t)\right\},
\end{aligned}
$$

for all $\beta>0$. Now let $\gamma, \lambda, \rho>0$, and note that

$$
\int_{0}^{\infty} s^{\rho-1} e^{-(\gamma+\lambda t) s} d s=\frac{\Gamma(\rho)}{(\gamma+\lambda t)^{\rho}},
$$

where $\Gamma(\cdot)$ denotes the standard gamma function [see 2, Chap. 6]. Then (7) follows from

$$
\begin{array}{r}
\mathbb{E}_{x}\left[\left(\gamma+\lambda \tau_{z}\right)^{-\rho}\right]=\int_{0}^{\infty} \frac{p_{z}(x, t)}{(\gamma+\lambda t)^{\rho}} d t=\int_{0}^{\infty} p_{z}(x, t) \frac{1}{\Gamma(\rho)} \int_{0}^{\infty} s^{\rho-1} e^{-(\gamma+\lambda t) s} d s d t \\
=\frac{1}{\Gamma(\rho)} \int_{0}^{\infty} e^{-\gamma s} s^{\rho-1} \int_{0}^{\infty} e^{-\lambda s t} p_{z}(x, t) d t d s=\frac{1}{\Gamma(\rho)} \mathscr{L}_{\gamma}\left\{s^{\rho-1} \mathscr{L}_{\lambda s}\left\{p_{z}(x, t)\right\}\right\} .
\end{array}
$$


A similar argument, using (9) again, gives

$$
\begin{aligned}
\mathscr{L}_{\alpha}\left\{\mathbb{E}_{x}\left[\mathbb{I}_{\left\{\tau_{z} \leq t\right\}}\left(\gamma+\lambda \tau_{z}\right)^{-\rho}\right]\right\}=\mathscr{L}_{\alpha}\left\{\int_{0}^{t} \frac{p_{z}(x, s)}{(\gamma+\lambda s)^{\rho}} d s\right\}=\frac{1}{\alpha} \mathscr{L}_{\alpha}\left\{\frac{p_{z}(x, t)}{(\gamma+\lambda t)^{\rho}}\right\} \\
=\frac{1}{\alpha} \int_{0}^{\infty} e^{-\alpha t} \frac{p_{z}(x, t)}{(\gamma+\lambda t)^{\rho}} d t=\frac{1}{\alpha} \int_{0}^{\infty} e^{-\alpha t} p_{z}(x, t) \frac{1}{\Gamma(\rho)} \int_{0}^{\infty} s^{\rho-1} e^{-(\gamma+\lambda t) s} d s d t \\
=\frac{1}{\alpha \Gamma(\rho)} \int_{0}^{\infty} e^{-\gamma s} s^{\rho-1} \int_{0}^{\infty} e^{-(\alpha+\lambda s) t} p_{z}(x, t) d t d s \\
=\frac{1}{\alpha \Gamma(\rho)} \mathscr{L}_{\gamma}\left\{s^{\rho-1} \mathscr{L}_{\alpha+\lambda s}\left\{p_{z}(x, t)\right\}\right\},
\end{aligned}
$$

which leads to $(8)$.

We note that (6) was obtained by Davidov and Linetsky [7, Prop. 2] by an application of Fubini's theorem. Also, (7) and (8) may be regarded as instances of the representation of generalized Stieltjes transforms as iterated Laplace transforms.

Next, suppose that $t>0$ and either $x, y \leq z$ or $x, y \geq z$. Combining (3) with (4) then yields

$$
\begin{aligned}
\tilde{G}_{\alpha}^{z}(x, y): & \mathscr{L}_{\alpha}\left\{\tilde{q}_{z}(t, x, y)\right\}=\mathscr{L}_{\alpha}\{q(t, x, y)\}-\mathscr{L}_{\alpha}\left\{p_{z}(x, t)\right\} \mathscr{L}_{\alpha}\{q(t, z, y)\} \\
= & \left\{\begin{array}{l}
G_{\alpha}(x, y)-\frac{\psi_{\alpha}(x)}{\psi_{\alpha}(z)} G_{\alpha}(z, y) \quad \text { if } x, y \leq z ; \\
G_{\alpha}(x, y)-\frac{\phi_{\alpha}(x)}{\phi_{\alpha}(z)} G_{\alpha}(z, y) \quad \text { if } x, y \geq z
\end{array}\right. \\
= & \begin{cases}w_{\alpha}^{-1} \psi_{\alpha}(x)\left(\phi_{\alpha}(y)-\frac{\phi_{\alpha}(z)}{\psi_{\alpha}(z)} \psi_{\alpha}(y)\right) \quad \text { if } x \leq y \leq z ; \\
w_{\alpha}^{-1} \psi_{\alpha}(y)\left(\phi_{\alpha}(x)-\frac{\phi_{\alpha}(z)}{\psi_{\alpha}(z)} \psi_{\alpha}(x)\right) \quad \text { if } y \leq x \leq z ; \\
w_{\alpha}^{-1}\left(\psi_{\alpha}(x)-\frac{\psi_{\alpha}(z)}{\phi_{\alpha}(z)} \phi_{\alpha}(x)\right) \phi_{\alpha}(y) \quad \text { if } y \geq x \geq z ; \\
w_{\alpha}^{-1}\left(\psi_{\alpha}(y)-\frac{\psi_{\alpha}(z)}{\phi_{\alpha}(z)} \phi_{\alpha}(y)\right) \phi_{\alpha}(x) \quad \text { if } x \geq y \geq z\end{cases}
\end{aligned}
$$

We have thus established the following result:

Lemma 3. The fundamental increasing and decreasing solutions to (2) corresponding to a lower killing boundary for $X$ at $a \in I$ are

$$
\tilde{\psi}_{\alpha}^{a}(x):=\psi_{\alpha}(x)-\frac{\psi_{\alpha}(a)}{\phi_{\alpha}(a)} \phi_{\alpha}(x) \quad \text { and } \quad \tilde{\phi}_{\alpha}^{a}(x):=\phi_{\alpha}(x),
$$

respectively, for all $x \in I \cap[a, \infty)$. The fundamental increasing and decreasing solutions to (2) corresponding to an upper killing boundary for $X$ at $b \in I$ are

$$
\tilde{\psi}_{\alpha}^{b}(x):=\psi_{\alpha}(x) \quad \text { and } \quad \tilde{\phi}_{\alpha}^{b}(x):=\phi_{\alpha}(x)-\frac{\phi_{\alpha}(b)}{\psi_{\alpha}(b)} \psi_{\alpha}(x),
$$

respectively, for all $x \in I \cap(-\infty, b]$. Finally, if the process is killed upon reaching either boundary, where $a<b$, then the relevant solutions to $(2)$ are $\tilde{\psi}_{\alpha}^{a, b}(\cdot):=\tilde{\psi}_{\alpha}^{a}(\cdot)$ and $\tilde{\phi}_{\alpha}^{a, b}(\cdot):=\tilde{\phi}_{\alpha}^{b}(\cdot)$. In each case the Laplace transform of the transition density of the killed diffusion is given by (1), with the appropriate functions replacing $\psi_{\alpha}(\cdot)$ and $\phi_{\alpha}(\cdot)$.

As an immediate consequence of the above lemma, we can now extend (4) as follows (see Davidov and Linetsky [7, Prop. 1] for an alternative proof): 
Proposition 4. Suppose $a, b \in I$ satisfy $a<b$. Then

$$
\begin{aligned}
& \mathbb{E}_{x}\left[\mathbb{I}_{\left\{\tau_{a}<\tau_{b}\right\}} e^{-\alpha \tau_{a}}\right]= \begin{cases}\frac{\psi_{\alpha}(x)}{\psi_{\alpha}(a)} & \text { if } x \leq a ; \\
\frac{\phi_{\alpha}(x) \psi_{\alpha}(b)-\psi_{\alpha}(x) \phi_{\alpha}(b)}{\phi_{\alpha}(a) \psi_{\alpha}(b)-\psi_{\alpha}(a) \phi_{\alpha}(b)} & \text { if } a \leq x \leq b ; \\
0 & \text { if } x \geq b,\end{cases} \\
& \mathbb{E}_{x}\left[\mathbb{I}_{\left\{\tau_{a}>\tau_{b}\right\}} e^{-\alpha \tau_{b}}\right]= \begin{cases}0 & \text { if } x \leq a ; \\
\frac{\phi_{\alpha}(a) \psi_{\alpha}(x)-\psi_{\alpha}(a) \phi_{\alpha}(x)}{\phi_{\alpha}(a) \psi_{\alpha}(b)-\psi_{\alpha}(a) \phi_{\alpha}(b)} & \text { if } a \leq x \leq b ; \\
\frac{\phi_{\alpha}(x)}{\phi_{\alpha}(b)} & \text { if } x \geq b,\end{cases}
\end{aligned}
$$

and

$$
\mathbb{E}_{x}\left[e^{-\alpha\left(\tau_{a} \wedge \tau_{b}\right)}\right]= \begin{cases}\frac{\psi_{\alpha}(x)}{\psi_{\alpha}(a)} & \text { if } x \leq a ; \\ \frac{\phi_{\alpha}(x)\left(\psi_{\alpha}(b)-\psi_{\alpha}(a)\right)-\psi_{\alpha}(x)\left(\phi_{\alpha}(b)-\phi_{\alpha}(a)\right)}{\phi_{\alpha}(a) \psi_{\alpha}(b)-\psi_{\alpha}(a) \phi_{\alpha}(b)} & \text { if } a \leq x \leq b ; \\ \frac{\phi_{\alpha}(x)}{\phi_{\alpha}(b)} & \text { if } x \geq b,\end{cases}
$$

for all $x \in I$.

Proof. To derive (13), let $x \in I$. The case when $x \geq b$ is obvious, so assume that $x<b$. Notice that $\tilde{\tau}_{a}^{b}:=\mathbb{I}_{\left\{\tau_{a}<\tau_{b}\right\}} \tau_{a}+\mathbb{I}_{\left\{\tau_{a}>\tau_{b}\right\}} \infty$ is the first-passage time to $a$ for the diffusion which is killed at $b$. Thus

$$
\mathbb{E}_{x}\left[\mathbb{I}_{\left\{\tau_{a}<\tau_{b}\right\}} e^{-\alpha \tau_{a}}\right]=\mathbb{E}_{x}\left[e^{-\alpha \tilde{\tau}_{a}^{b}}\right]= \begin{cases}\frac{\tilde{\psi}_{\alpha}^{b}(x)}{\tilde{\psi}_{\alpha}^{b}(a)} & \text { if } x \leq a \\ \frac{\tilde{\phi}_{\alpha}^{b}(x)}{\tilde{\phi}_{\alpha}^{b}(a)} & \text { if } x \leq a,\end{cases}
$$

according to (4). The result follows by substituting (12) into the expressions above. The derivation of (14) is similar, while (15) follows by adding (13) and (14).

We end this section by analyzing reflecting boundaries. Let $\hat{q}^{z}(\cdot, \cdot, \cdot)$ denote the transition density (with respect to speed measure) of $X$, with reflection at $z \in I$. Since the reflected diffusion is still a Markov process, the same argument as in Lemma 1 gives

$$
\hat{q}^{z}(t, x, y)=\tilde{q}_{z}(t, x, y)+\int_{0}^{t} p_{z}(x, s) \hat{q}^{z}(t-s, z, y) d s,
$$

for all $x, y \in I$ with $x, y \leq z$ or $x, y \geq z$, and all $t>0$. The reflecting boundary condition may be expressed as $\left.\frac{\partial}{\partial x} \hat{q}^{z}(t, x, y)\right|_{x=z}=0$ [see e.g. 17, p. 332], and so we obtain the following result from (16), by computing Laplace transforms and differentiating, before applying (4) and (10):

$$
\begin{aligned}
\mathscr{L}_{\alpha}\left\{\hat{q}^{z}(t, z, y)\right\}= & -\frac{\left.\frac{\partial}{\partial x} \mathscr{L}_{\alpha}\left\{\tilde{q}_{z}(t, x, y)\right\}\right|_{x=z}}{\left.\frac{\partial}{\partial x} \mathscr{L}_{\alpha}\left\{p_{z}(x, t)\right\}\right|_{x=z}} \\
& = \begin{cases}w_{\alpha}^{-1} \psi_{\alpha}(y)\left(\phi_{\alpha}(z)-\frac{\phi_{\alpha}^{\prime}(z)}{\psi_{\alpha}^{\prime}(z)} \psi_{\alpha}(z)\right) & \text { if } y \leq z ; \\
w_{\alpha}^{-1}\left(\psi_{\alpha}(z)-\frac{\psi_{\alpha}^{\prime}(z)}{\phi_{\alpha}^{\prime}(z)} \phi_{\alpha}(z)\right) \phi_{\alpha}(y) & \text { if } y \geq z .\end{cases}
\end{aligned}
$$


Combining the above with (16) yields

$$
\begin{aligned}
\hat{G}_{\alpha}^{z}(x, y): & \mathscr{L}_{\alpha}\left\{\hat{q}^{z}(t, x, y)\right\}=\mathscr{L}_{\alpha}\left\{\tilde{q}_{z}(t, x, y)\right\}+\mathscr{L}_{\alpha}\left\{p_{z}(x, t)\right\} \mathscr{L}_{\alpha}\left\{\hat{q}^{z}(t, z, y)\right\} \\
= & \left\{\begin{array}{l}
\tilde{G}_{\alpha}^{z}(x, y)-w_{\alpha}^{-1} \psi_{\alpha}(y)\left(\phi_{\alpha}(z)-\frac{\phi_{\alpha}^{\prime}(z)}{\psi_{\alpha}^{\prime}(z)} \psi_{\alpha}(z)\right) \frac{\psi_{\alpha}(x)}{\psi_{\alpha}(z)} \quad \text { if } x, y \leq z ; \\
\tilde{G}_{\alpha}^{z}(x, y)-w_{\alpha}^{-1}\left(\psi_{\alpha}(z)-\frac{\psi_{\alpha}^{\prime}(z)}{\phi_{\alpha}^{\prime}(z)} \phi_{\alpha}(z)\right) \phi_{\alpha}(y) \frac{\phi_{\alpha}(x)}{\phi_{\alpha}(z)} \quad \text { if } x, y \geq z
\end{array}\right. \\
= & \begin{cases}w_{\alpha}^{-1} \psi_{\alpha}(x)\left(\phi_{\alpha}(y)-\frac{\phi_{\alpha}^{\prime}(z)}{\psi_{\alpha}^{\prime}(z)} \psi_{\alpha}(y)\right) \quad \text { if } x \leq y \leq z ; \\
w_{\alpha}^{-1} \psi_{\alpha}(y)\left(\phi_{\alpha}(x)-\frac{\phi_{\alpha}^{\prime}(z)}{\psi_{\alpha}^{\prime}(z)} \psi_{\alpha}(x)\right) \quad \text { if } y \leq x \leq z ; \\
w_{\alpha}^{-1}\left(\psi_{\alpha}(x)-\frac{\psi_{\alpha}^{\prime}(z)}{\phi_{\alpha}^{\prime}(z)} \phi_{\alpha}(x)\right) \phi_{\alpha}(y) \quad \text { if } y \geq x \geq z ; \\
w_{\alpha}^{-1}\left(\psi_{\alpha}(y)-\frac{\psi_{\alpha}^{\prime}(z)}{\phi_{\alpha}^{\prime}(z)} \phi_{\alpha}(y)\right) \phi_{\alpha}(x) \quad \text { if } x \geq y \geq z .\end{cases}
\end{aligned}
$$

We may express this formally as follows:

Lemma 5. The fundamental increasing and decreasing solutions to (2) corresponding to a lower reflecting boundary for $X$ at $a \in I$ are

$$
\hat{\psi}_{\alpha}^{a}(x):=\psi_{\alpha}(x)-\frac{\psi_{\alpha}^{\prime}(a)}{\phi_{\alpha}^{\prime}(a)} \phi_{\alpha}(x) \quad \text { and } \quad \hat{\phi}_{\alpha}^{a}(x):=\phi_{\alpha}(x),
$$

respectively, for all $x \in I \cap[a, \infty)$. The fundamental increasing and decreasing solutions to (2) corresponding to an upper reflecting boundary for $X$ at $b \in I$ are

$$
\hat{\psi}_{\alpha}^{b}(x):=\psi_{\alpha}(x) \quad \text { and } \quad \hat{\phi}_{\alpha}^{b}(x):=\phi_{\alpha}(x)-\frac{\phi_{\alpha}^{\prime}(b)}{\psi_{\alpha}^{\prime}(b)} \psi_{\alpha}(x),
$$

respectively, for all $x \in I \cap(-\infty, b]$. Finally, if the process is reflected at either boundary, where $a<b$, then the relevant solutions to $(2)$ are $\hat{\psi}_{\alpha}^{a, b}(\cdot):=\hat{\psi}_{\alpha}^{a}(\cdot)$ and $\hat{\phi}_{\alpha}^{a, b}(\cdot):=\hat{\phi}_{\alpha}^{b}(\cdot)$. In each case the Laplace transform of the transition density of the reflected diffusion is given by (1), with the appropriate functions replacing $\psi_{\alpha}(\cdot)$ and $\phi_{\alpha}(\cdot)$.

It should be noted that the results of Lemma 3 and Lemma 5 can be obtained by inspection. This is because the boundary behaviour of the diffusion determines the boundary conditions that must be imposed on the fundamental solutions of (2). Since the monotone increasing and decreasing solutions that satisfy these boundary conditions will be unique (up to a multiplicative constant), we have enough information to identify them [see 4, pp. 18-19].

3. Squared Bessel processes. Suppose now that $X=\left(X_{t}\right)_{t \geq 0}$ is a squared Bessel process of dimension $\delta \in \mathbb{R}$ [see 14,25$]$. For any $x \in I$, this process is a strong solution of the following stochastic differential equation (SDE) under $\mathbb{P}_{x}$ :

$$
X_{t}=x+\delta t+2 \int_{0}^{t} \sqrt{X_{s}} d W_{s}
$$

for all $t \geq 0$, where $W=\left(W_{t}\right)_{t \geq 0}$ is a standard Brownian motion. Its infinitesimal generator is given by

$$
\mathcal{G} f(x):=2 x f^{\prime \prime}(x)+\delta f^{\prime}(x)
$$


for all suitable functions $f(\cdot)$ and all $x \in I$, while its scale function and speed measure are given by

$$
\mathfrak{s}(x):=\left\{\begin{array}{ll}
\frac{2}{2-\delta} x^{\frac{2-\delta}{2}} & \text { if } \delta \neq 2 ; \\
\ln x & \text { if } \delta=2
\end{array} \quad \text { and } \quad \mathfrak{m}(x):=\frac{1}{2} x^{\frac{\delta-2}{2}},\right.
$$

respectively, for all $x \in I$.

Feller's test indicates that this process has a natural boundary at infinity, and a boundary at the origin which is absorbing if $\delta \leq 0$; natural if $\delta \geq 2$; and regular if $0<\delta<2$. In the latter case, the behaviour of $X$ at zero must be specified: If the origin is a killing boundary, then $I=(0, \infty)$; while $I=[0, \infty)$ if it is a reflecting boundary. The following fundamental solutions to (2) are provided by Borodin and Salminen [4, p. 135]:

$$
\psi_{\alpha}(x)= \begin{cases}x^{\frac{2-\delta}{4}} I_{\frac{\delta-2}{2}}(\sqrt{2 \alpha x}) & \text { if } \delta \geq 2 \text { or } 0<\delta<2 \text { and } 0 \text { is reflecting } \\ x^{\frac{2-\delta}{4}} I_{\frac{2-\delta}{2}}(\sqrt{2 \alpha x}) & \text { if } \delta \leq 0 \text { or } 0<\delta<2 \text { and } 0 \text { is killing }\end{cases}
$$

and

$$
\phi_{\alpha}(x)=x^{\frac{2-\delta}{4}} K_{\frac{\delta-2}{2}}(\sqrt{2 \alpha x}),
$$

for all $\alpha>0$ and $x \geq 0$. Here $I_{\nu}(\cdot)$ and $K_{\nu}(\cdot)$ denote the modified Bessel functions, with index $\nu$, of the first and second kinds, respectively [see 2, Chap. 9]. The associated Wronskian is $w_{\alpha}=1 / 2$.

The recognized authority on squared Bessel processes is Revuz and Yor [25, Chap. XI], where reflection at zero is the default boundary condition, for all dimensions $0<\delta<2$. In this case one obtains the following transition density (with respect to speed measure), for all $\delta>0$ :

$$
q(t, x, y)= \begin{cases}\frac{1}{t}(x y)^{\frac{2-\delta}{4}} e^{-\frac{x+y}{2 t}} I_{\frac{\delta-2}{2}\left(\frac{\sqrt{x y}}{t}\right)} & \text { if } x>0 \\ \frac{2}{(2 t)^{\delta / 2} \Gamma(\delta / 2)} e^{-\frac{y}{2 t}} & \text { if } x=0\end{cases}
$$

for all $t>0$ and $x, y \geq 0$. In the case when $0<\delta<2$, Borodin and Salminen [4, p. 136] found the transition density of the squared Bessel process killed at the origin, by Laplace transform inversion of the appropriate Green's function. However, Göing-Jaeschke and Yor [14] derived the following explicit density for the first-passage time to zero of $X$, when $\delta<2$ :

$$
p_{0}(x, t)=\frac{1}{t \Gamma\left(\frac{2-\delta}{2}\right)}\left(\frac{x}{2 t}\right)^{\frac{2-\delta}{2}} e^{-\frac{x}{2 t}},
$$

for all $t>0$ and $x \geq 0$. Combining this with (20), we can derive the above-mentioned transition density, with killing at the origin, directly from (3), without the need to invert a Laplace transform. We start with a technical lemma, expressing the modified Bessel function of the second kind as an indefinite integral:

Lemma 6. Let $\nu \in \mathbb{R}$ be arbitrary. Then

$$
K_{\nu}(z)=\frac{1}{2} \int_{0}^{\infty} t^{\nu-1} e^{-\frac{1}{2}(t+1 / t) z} d t
$$

for all $z \in \mathbb{C}$ with $\Re z>0$. 
Proof. Starting with an identity for $K_{\nu}(\cdot)$ found in Lebedev [19, p. 119], we obtain

$$
\begin{aligned}
K_{\nu}(z) & =\int_{0}^{\infty} e^{-z \cosh u} \cosh \nu u d u \\
& =\frac{1}{2} \underbrace{\int_{0}^{\infty} e^{-z \cosh u} e^{\nu u} d u}_{I_{1}}+\frac{1}{2} \underbrace{\int_{0}^{\infty} e^{-z \cosh u} e^{-\nu u} d u}_{I_{2}}
\end{aligned}
$$

These integrals are evaluated as follows:

$$
I_{1}=\int_{1}^{\infty} t^{\nu-1} e^{-\frac{1}{2}(t+1 / t) z} d t \quad \text { and } \quad I_{2}=\int_{0}^{1} t^{\nu-1} e^{-\frac{1}{2}(t+1 / t) z} d t,
$$

with the help of the respective substitutions $e^{u} \mapsto t$ and $e^{-u} \mapsto t$.

Proposition 7. The transition density (with respect to speed measure) of the squared Bessel process of dimension $0<\delta<2$, killed at the origin, is given by

$$
\tilde{q}_{0}(t, x, y)=\frac{1}{t}(x y)^{\frac{2-\delta}{4}} e^{-\frac{x+y}{2 t}} I_{\frac{2-\delta}{2}}\left(\frac{\sqrt{x y}}{t}\right),
$$

for all $t>0$ and $x, y \geq 0$.

Proof. It follows from (3), (20) and (21) that

$$
\begin{aligned}
q(t, x, y)-\tilde{q}_{0}(t, x, y) & =\int_{0}^{t} p_{0}(x, s) q(t-s, 0, y) d s \\
& =\frac{4}{\Gamma(\delta / 2) \Gamma\left(\frac{2-\delta}{2}\right)} x^{\frac{2-\delta}{2}} \int_{0}^{t} \frac{e^{-\frac{x}{2 s}-\frac{y}{2(t-s)}}}{(2 s)^{\frac{4-\delta}{2}}(2(t-s))^{\delta / 2}} d s \\
& =\frac{4}{\pi} \sin \left(\frac{\delta}{2} \pi\right) x^{\frac{2-\delta}{2}} \int_{0}^{t} \frac{e^{-\frac{x}{2 s}-\frac{y}{2(t-s)}}}{(2 s)^{\frac{4-\delta}{2}}(2(t-s))^{\delta / 2}} d s .
\end{aligned}
$$

For the final equality above, we use the reflection formula $\Gamma(z) \Gamma(1-z)=\frac{\pi}{\sin \pi z}$, which holds for all $z \in \mathbb{C} \backslash \mathbb{Z}$ [see e.g. 19, p. 3]. Continuing, with the aid of the transformation $(t / s-1) \sqrt{x / y} \mapsto \zeta$, we obtain

$$
\begin{aligned}
q(t, x, y)-\tilde{q}_{0}(t, x, y) & =\frac{1}{\pi} \sin \left(\frac{\delta}{2} \pi\right) \frac{1}{t}(x y)^{\frac{2-\delta}{4}} e^{-\frac{x+y}{2 t}} \int_{0}^{\infty} \frac{e^{-\frac{1}{2}(\zeta+1 / \zeta) \frac{\sqrt{x y}}{t}}}{\zeta^{\delta / 2}} d \zeta \\
& =\frac{2}{\pi} \sin \left(\frac{\delta}{2} \pi\right) \frac{1}{t}(x y)^{\frac{2-\delta}{4}} e^{-\frac{x+y}{2 t}} K_{\frac{2-\delta}{2}}\left(\frac{\sqrt{x y}}{t}\right)
\end{aligned}
$$

from (22). Using the relation $K_{\nu}(z)=\frac{\pi}{2} \frac{I_{-\nu}(z)-I_{\nu}(z)}{\sin \nu \pi}$, which holds if $|\arg z|<\pi$ and $\nu \notin \mathbb{Z}$ [see e.g. 19, p. 108], and bearing in mind that $\sin \frac{2-\delta}{2} \pi=\sin \frac{\delta}{2} \pi$, we finally get

$$
q(t, x, y)-\tilde{q}_{0}(t, x, y)=\frac{1}{t}(x y)^{\frac{2-\delta}{4}} e^{-\frac{x+y}{2 t}}\left[I_{\frac{\delta-2}{2}}\left(\frac{\sqrt{x y}}{t}\right)-I_{\frac{2-\delta}{2}}\left(\frac{\sqrt{x y}}{t}\right)\right] .
$$

The desired result follows by subtracting (20) from both sides of this equation.

4. The minimal market model. Let $\mathbb{P}_{t, S}$ denote the probability measure under which a global diversified equity index $S^{*}=\left(S_{t+u}^{*}\right)_{u \geq 0}$ starts at time $t \geq 0$ with value $S>0$; 
we shall on occasion refer to it as the real-world measure. The dynamics of the index under this measure are expressed by the following SDE:

$$
S_{t+u}^{*}=S+\int_{0}^{u}\left(r+\vartheta^{2}\left(t+v, S_{t+v}^{*}\right)\right) S_{t+v}^{*} d v+\int_{0}^{u} \vartheta\left(t+v, S_{t+v}^{*}\right) S_{t+v}^{*} d W_{v},
$$

for all $u \geq 0$. Here $r \geq 0$ is a constant risk-free interest rate, while $W=\left(W_{u}\right)_{u \geq 0}$ is a standard Brownian motion starting at zero under $\mathbb{P}_{t, S}$. Furthermore, $\vartheta(\cdot, \cdot)$ is a local volatility function, given by

$$
\vartheta(t, S):=\sqrt{\frac{\alpha e^{(r+\eta) t}}{S}},
$$

for all $t \geq 0$ and $S>0$, where $\alpha, \eta>0$ are fixed parameters.

Together, (24) and (25) constitute a model for a global diversified portfolio, called the minimal market model (MMM). Among its attractive features, it captures the observed inverse relationship between price and volatility, dubbed the "leverage effect" by Black [3]. It is also relatively parsimonious, with only two free parameters. We must stress that the MMM is intended as a description of the observable real-world behaviour of the index - in contradistinction to most literature on stochastic finance, we are not concerned with riskneutral dynamics. A detailed study of the model is presented in Platen and Heath [24, Chap. 13]. We should point out that what we have described here is in fact a slightly simplified version of the general model, referred to as the "stylized" version by Platen and Heath [24, Sec. 13.2]. In general, (24) may contain a third parameter, describing risk aversion.

For any $t \geq 0$ and $S>0$, let $X=\left(X_{u}\right)_{u \geq 0}$ henceforth be a squared Bessel process of dimension four, starting at $e^{-r t} S$ under $\mathbb{P}_{t, S}$. Using Itô's formula, we obtain the following representation for $S^{*}$ under this probability measure:

$$
S_{t+u}^{*} \stackrel{(\mathrm{d})}{=} e^{r(t+u)} X_{\varphi_{t}(u)},
$$

for all $u \geq 0$. Here $\varphi_{t}(\cdot)$ is a deterministic time transform, given by

$$
\varphi_{t}(u):=\frac{\alpha}{4 \eta} e^{\eta t}\left(e^{\eta u}-1\right),
$$

for all $u \geq 0$. The importance of (26) lies in the fact that the transition density (20) of $X$ is known explicitly. Consequently, algebraic expressions can often be derived for the expected values of functionals of $S^{*}$. This is particularly relevant for obtaining pricing formulae for contingent claims written on the index.

In addition to the index, we assume that the market also contains a risk-free savings account. Under the probability measure $\mathbb{P}_{t, S}$, with $t \geq 0$ and $S>0$, this is a deterministic process $B=\left(B_{t+u}\right)_{u \geq 0}$, given by $B_{t+u}:=e^{r(t+u)}$, for all $u \geq 0$. Although the MMM does not admit an equivalent risk-neutral probability measure [see 24, pp. 499-500], it is nevertheless the case that $S^{*}$ is a numéraire for $\mathbb{P}_{t, S}$, in the sense that all self-financing portfolios comprising $B$ and $S^{*}$ are $\mathbb{P}_{t, S}$-local martingales, when denominated in units of $S^{*}$ [see e.g. 13]. This has two important consequences: Firstly, it means that the MMM is free of economically meaningful arbitrage opportunities [see 20, 24, p. 376]. Secondly, it paves the way for a martingale approach to contingent claim pricing under $\mathbb{P}_{t, S}$, called "real-world pricing" [see 24, pp. 325-326]. 
To understand contingent claim pricing with the MMM, let $\mathbf{F}^{W}=\left(\mathscr{F}_{u}^{W}\right)_{u \geq 0}$ denote the filtration generated by the Brownian motion $W$, suppose that $\tau$ is an $\mathbf{F}^{W}$-stopping time, and let $h(\cdot)$ be an appropriate Borel-measurable payoff function. The real-world price at time $t \geq 0$ of the claim $h\left(S_{t+\tau}^{*}\right) \in L^{1}\left(\mathscr{F}_{\tau}^{W}\right)$, maturing at time $t+\tau$, is then given by

$$
V^{h}(t, S):=S \mathbb{E}_{t, S}\left[\mathbb{I}_{\{\tau<\infty\}} \frac{h\left(S_{t+\tau}^{*}\right)}{S_{t+\tau}^{*}}\right],
$$

when $S_{t}^{*}=S>0$. It is important to remember that here $\mathbb{E}_{t, S}$ is the expected value operator with respect to the real-world probability measure $\mathbb{P}_{t, S}$.

Obviously, the indicator function in (28) may be omitted if $\tau<\infty$ a.s. It may also be dropped if $h(\cdot)$ is bounded, since (26) and the transience of a squared Bessel process of dimension $\delta \geq 3$ [see 25 , p. 442] together imply that $\lim _{t \rightarrow \infty} S_{t}^{*}=\infty$ a.s. As mentioned before, (26) and (20) often allow us to derive the pricing function $V^{h}(\cdot, \cdot)$ explicitly.

5. Rebates. We now consider the valuation of a rebate written on the index. This is a claim that pays $\$ 1$ as soon as the index hits a certain level, provided this occurs before a contracted expiry date $T>0$. In our case the trigger level for the rebate is a deterministic barrier $Z=\left(Z_{t}\right)_{t \geq 0}$, with $Z_{t}:=z e^{r t}$, for some $z>0$. The fact that it grows at the riskfree rate is economically quite attractive, since it makes the price of the rebate sensitive to the performance of the index relative to that of the savings account. This feature is particularly desirable for long-dated instruments, due to the observed long-term growth of diversified equity portfolios. The probability of such a portfolio reaching a predetermined fixed level in the future becomes increasingly remote, with the passage of time.

We start by introducing the stopping times

$$
\sigma_{z, t}:=\inf \left\{u>0 \mid S_{t+u}^{*}=Z_{t+u}\right\} \quad \text { and } \quad \tau_{z}:=\inf \left\{u>0 \mid X_{u}=z\right\},
$$

for any $t \geq 0$. It then follows from (26) and (27) that

$$
\sigma_{z, t}=\inf \left\{u>0 \mid X_{\varphi_{t}(u)}=z\right\} \stackrel{(\mathrm{d})}{=} \varphi_{t}^{-1}\left(\tau_{z}\right)=\frac{1}{\eta} \ln \left(1+\frac{4 \eta}{\alpha} e^{-\eta t} \tau_{z}\right) .
$$

First, we consider the valuation of a perpetual rebate, for which $T=\infty$. Using (28), (29) and $(7)$, the pricing function $\mathrm{R}_{\infty, z}(\cdot, \cdot)$ for this instrument is given by

$$
\begin{aligned}
& \mathrm{R}_{\infty, z}(t, S)=S \mathbb{E}_{t, S}\left[\frac{1}{S_{t+\sigma_{z, t}}^{*}}\right]=\frac{e^{-r t} S}{z} \mathbb{E}_{t, S}\left[e^{-r \sigma_{z, t}}\right] \\
& =\frac{e^{-r t} S}{z} \mathbb{E}_{t, S}\left[e^{-r \varphi_{t}^{-1}\left(\tau_{z}\right)}\right]=\frac{e^{-r t} S}{z} \mathbb{E}_{t, S}\left[\left(1+\frac{4 \eta}{\alpha} e^{-\eta t} \tau_{z}\right)^{-r / \eta}\right] \\
& = \begin{cases}\frac{x}{z} \frac{1}{\Gamma(r / \eta)} \int_{0}^{\infty} e^{-s} s^{r / \eta-1} \frac{\psi_{4 \eta / \alpha e^{-\eta t_{s}}}(x)}{\psi_{4 \eta / \alpha e^{-\eta t_{s}}}(z)} d s & \text { if } x \leq z ; \\
\frac{x}{z} \frac{1}{\Gamma(r / \eta)} \int_{0}^{\infty} e^{-s} s^{r / \eta-1} \frac{\phi_{4 \eta / \alpha e^{-\eta t_{s}}}(x)}{\phi_{4 \eta / \alpha e^{-\eta t_{s}}}(z)} d s & \text { if } x \geq z,\end{cases}
\end{aligned}
$$

for all $t \geq 0$ and $S>0$, with $x:=e^{-r t} S$ in the final line, for convenience.

We may test the validity of the above pricing formula by examining a special case. Suppose for a moment that $\sigma_{z, t}=0$, implying that the rebate pays immediately under $\mathbb{P}_{t, S}$. This only happens if $S=Z_{t}=z e^{r t}$, which in turn means that $x=z$. It then follows 

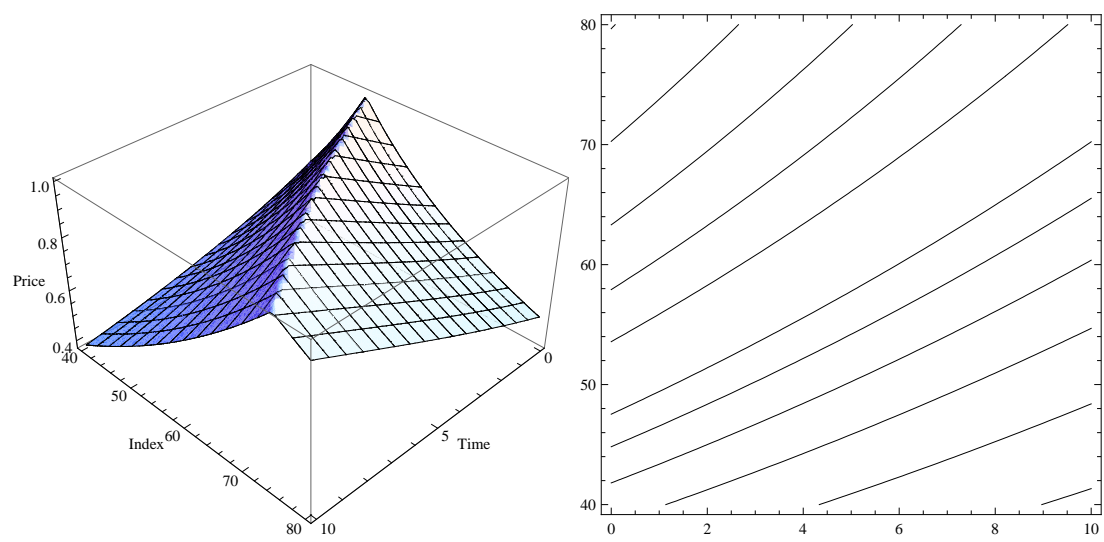

Fig. 1. The perpetual rebate pricing function $R_{\infty, 50}(\cdot, \cdot)$

from (30), together with the definition of the gamma function, that $\mathrm{R}_{\infty, z}(t, S)=1$, as expected.

Computing a perpetual rebate price with (30) necessarily involves numerical quadrature. This is not a significant obstacle to using the formula, since numerous quick and accurate schemes exist for one-dimensional quadrature problems - we have simply used the NIntegrate[...] function in Mathematica 6 . To assist with numerical evaluation, we do, however, recommend first transforming the domain of integration into a finite interval, via the change of variables $e^{-s} \mapsto u$. This results in the following pricing formula:

$$
\mathrm{R}_{\infty, z}(t, S)= \begin{cases}\frac{x}{z} \frac{1}{\Gamma(r / \eta)} \int_{0}^{1}(-\ln u)^{r / \eta-1} \frac{\psi_{-4 \eta / \alpha e^{-\eta t} \ln u}(x)}{\psi_{-4 \eta / \alpha e^{-\eta t} \ln u}(z)} d u & \text { if } x \leq z ; \\ \frac{x}{z} \frac{1}{\Gamma(r / \eta)} \int_{0}^{1}(-\ln u)^{r / \eta-1} \frac{\phi_{-4 \eta / \alpha e^{-\eta t} \ln u}(x)}{\phi_{-4 \eta / \alpha e^{-\eta t} \ln u}(z)} d u & \text { if } x \geq z\end{cases}
$$

with $x:=e^{-r t} S$, as before.

Figure 1 presents surface and contour plots of the pricing function $R_{\infty, 50}(\cdot, \cdot)$ for a perpetual rebate with reference level $z=50$, by numerical integration of (31). The parameter values used for the graphs were $\alpha=1, \eta=0.05$ and $r=0.04$. These are reasonably close to values estimated from historical data for the S\&P500 index.

We turn our attention now to the rebate with finite maturity $T<\infty$. It follows from (28) and (29) that the pricing function $\mathrm{R}_{T, z}(\cdot, \cdot)$ of this claim is determined by

$$
\begin{aligned}
\mathrm{R}_{T, z}(t, S) & =S \mathbb{E}_{t, S}\left[\frac{\mathbb{I}_{\left\{t+\sigma_{z, t} \leq T\right\}}}{S_{t+\sigma_{z, t}}^{*}}\right]=\frac{e^{-r t} S}{z} \mathbb{E}_{t, S}\left[\mathbb{I}_{\left\{\sigma_{z, t} \leq T-t\right\}} e^{-r \sigma_{z, t}}\right] \\
& =\frac{e^{-r t} S}{z} \mathbb{E}_{t, S}\left[\mathbb{I}_{\left\{\varphi_{t}^{-1}\left(\tau_{z}\right) \leq T-t\right\}} e^{-r \varphi_{t}^{-1}\left(\tau_{z}\right)}\right] \\
& =\frac{e^{-r t} S}{z} \mathbb{E}_{t, S}\left[\mathbb{I}_{\left\{\tau_{z} \leq \varphi_{t}(T-t)\right\}}\left(1+\frac{4 \eta}{\alpha} e^{-\eta t} \tau_{z}\right)^{-r / \eta}\right] .
\end{aligned}
$$

We now use (8) to compute the Laplace transform of (32), with respect to transformed 
time-to-maturity:

$$
\mathscr{L}_{\beta}\left\{\mathrm{R}_{T, z}(t, S)\right\}= \begin{cases}\frac{x}{z} \frac{1}{\beta \Gamma(r / \eta)} \int_{0}^{\infty} e^{-s} s^{r / \eta-1} \frac{\psi_{\beta+4 \eta / \alpha e^{-\eta t_{s}}}(x)}{\psi_{\beta+4 \eta / \alpha e^{-\eta t_{s}}(z)}} d s & \text { if } x \leq z \\ \frac{x}{z} \frac{1}{\beta \Gamma(r / \eta)} \int_{0}^{\infty} e^{-s} s^{r / \eta-1} \frac{\phi_{\beta+4 \eta / \alpha e^{-\eta t_{s}}}(x)}{\phi_{\beta+4 \eta / \alpha e^{-\eta t_{s}}}(z)} d s & \text { if } x \geq z\end{cases}
$$

for all $\beta>0$, with $x:=e^{-r t} S$.

Pricing a finite maturity rebate thus involves two numerical procedures: First the integral in (33) must be evaluated by quadrature, and then the Laplace transform itself must be inverted. For the numerical integration, we proceed as before, by first performing the substitution $e^{-s} \mapsto u$, so that (33) becomes

$$
\mathscr{L}_{\beta}\left\{\mathrm{R}_{T, z}(t, S)\right\}= \begin{cases}\frac{x}{z} \frac{1}{\beta \Gamma(r / \eta)} \int_{0}^{1}(-\ln u)^{r / \eta-1} \frac{\psi_{\beta-4 \eta / \alpha e^{-\eta t} \ln u}(x)}{\psi_{\beta-4 \eta / \alpha e^{-\eta t} \ln u}(z)} d u & \text { if } x \leq z \\ \frac{x}{z} \frac{1}{\beta \Gamma(r / \eta)} \int_{0}^{1}(-\ln u)^{r / \eta-1} \frac{\phi_{\beta-4 \eta / \alpha e^{-\eta t} \ln u}(x)}{\phi_{\beta-4 \eta / \alpha e^{-\eta t} \ln u}(z)} d u & \text { if } x \geq z .\end{cases}
$$

For the inversion of (34), we recommend the Euler method presented in Abate and Whitt [1]. This technique employs Euler summation to evaluate the Fourier inversion integral along the Bromwich contour, and has the advantage of being uncomplicated and quick. Although there are no guaranteed error bounds, the same method has been used successfully before, e.g. by Davidov and Linetsky [7, 8] and Fu et al. [10], to invert Laplace transforms associated with derivative valuation problems. We also draw the reader's attention to the comparative study of numerical schemes for Laplace transform inversion in Craddock et al. [6], which focused specifically on applications to derivative pricing.

Figure 2 presents surface and contour plots of the pricing function $\mathrm{R}_{10,50}(\cdot, \cdot)$ for a rebate with maturity $T=10$ years and reference level $z=50$, using the same parameter values as before. We see again that $\mathrm{R}_{T, z}\left(t, Z_{t}\right)=1$, as expected. Furthermore, we see that

$$
\lim _{t \rightarrow T} R_{T, z}(t, S)= \begin{cases}1 & \text { if } S=Z_{T} \\ 0 & \text { otherwise }\end{cases}
$$

This agrees with the economically obvious behaviour of the rebate price close to maturity.
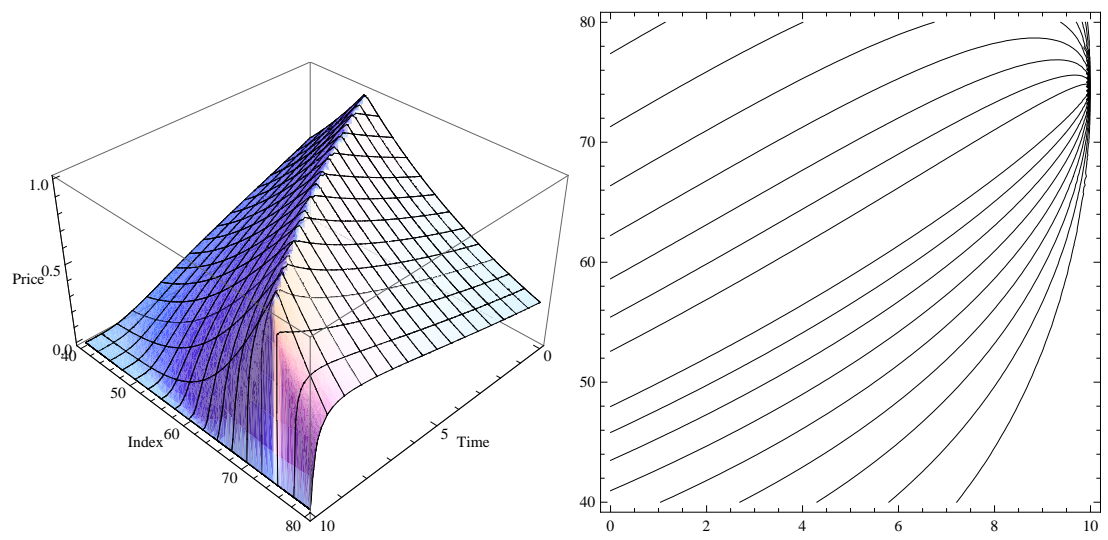

Fig. 2. The rebate pricing function $R_{10,50}(\cdot, \cdot)$ 
6. Barrier options. A barrier option written on the index is another example of a contingent claim whose payoff is determined by whether or not the index hits a certain level prior to its maturity $T>0$. In this section we consider a European call on the index, with strike price $K>0$, which is knocked out if the index breaches the same deterministic barrier $Z$ as in Section 5, sometime before expiry.

Starting with (28), and using (29), we derive the following expression for the real-world price of this instrument at time $t \in[0, T)$, given the starting index value $S_{t}^{*}=S>0$ :

$$
\begin{aligned}
\mathrm{C}_{T, K, z}^{\text {out }}(t, S) & =S \mathbb{E}_{t, S}\left[\mathbb{I}_{\left\{t+\sigma_{z, t}>T\right\}} \frac{\left(S_{T}^{*}-K\right)^{+}}{S_{T}^{*}}\right] \\
& =S \mathbb{E}_{t, S}\left[\mathbb{I}_{\left\{\sigma_{z, t}>T-t\right\}}\left(1-\frac{K}{S_{T}^{*}}\right)^{+}\right] \\
& =S \mathbb{E}_{t, S}\left[\mathbb{I}_{\left\{\tau_{z}>\varphi_{t}(T-t)\right\}}\left(1-\frac{e^{-r T} K}{X_{\varphi_{t}}(T-t)}\right)^{+}\right] \\
& =S \int_{e^{-r T} K}^{\infty}\left(1-\frac{e^{-r T} K}{y}\right) \tilde{q}_{z}\left(\varphi_{t}(T-t), e^{-r t} S, y\right) \mathfrak{m}(y) d y \\
& =\frac{1}{2} S \int_{\kappa}^{\infty}(y-\kappa) \tilde{q}_{z}\left(\varphi_{t}(T-t), x, y\right) d y
\end{aligned}
$$

where $x:=e^{-r t} S$ and $\kappa:=e^{-r T} K$. Recall also that the speed measure of a squared Bessel process of dimension four is given by $\mathfrak{m}(y):=y / 2$, according to (17). Computing the Laplace transform of (35), with respect to transformed time-to-maturity, now yields

$$
\begin{aligned}
\mathscr{L}_{\beta}\left\{\mathrm{C}_{T, K, z}^{\text {out }}(t, S)\right\}=\int_{0}^{\infty} e^{-\beta u}\left(\frac{1}{2} S \int_{\kappa}^{\infty}(y-\kappa) \tilde{q}_{z}(u, x, y) d y\right) d u \\
=\frac{1}{2} S \int_{\kappa}^{\infty}(y-\kappa) \mathscr{L}_{\beta}\left\{\tilde{q}_{z}(u, x, y)\right\} d y=\frac{1}{2} S \int_{\kappa}^{\infty}(y-\kappa) \tilde{G}_{\beta}^{z}(x, y) d y
\end{aligned}
$$

for all $\beta>0$.

For convenience, we now analyze the following two cases separately:

$$
\begin{aligned}
\text { up-and-out call: } & S \leq Z_{t}=z e^{r t} \text { iff } x \leq z ; \\
\text { down-and-out call: } & S \geq Z_{t}=z e^{r t} \text { iff } x \geq z .
\end{aligned}
$$

In the first case, we may truncate the integral in (36), since $x \leq z$ implies that $\tilde{G}_{\beta}^{z}(x, y)=$ 0 , for all $y \geq z$. Together with (10), this allows us to express the Laplace transform of the up-and-out call as follows:

$$
\begin{aligned}
\mathscr{L}_{\beta}\left\{\mathrm{C}_{T, K, z}^{\text {out }}(t, S)\right\}= & S \int_{\kappa}^{\kappa \vee x}(y-\kappa) \psi_{\beta}(y)\left(\phi_{\beta}(x)-\frac{\phi_{\beta}(z)}{\psi_{\beta}(z)} \psi_{\beta}(x)\right) d y \\
& +S \int_{\kappa \vee x}^{\kappa \vee z}(y-\kappa) \psi_{\beta}(x)\left(\phi_{\beta}(y)-\frac{\phi_{\beta}(z)}{\psi_{\beta}(z)} \psi_{\beta}(y)\right) d y,
\end{aligned}
$$

if $x \leq z$. In the second case, $x \geq z$ implies that $\tilde{G}_{\beta}^{z}(x, y)=0$, for all $y \leq z$. Combining this with (10) produces the following expression for the Laplace transform of the down-and-out 
call:

$$
\begin{aligned}
\mathscr{L}_{\beta}\left\{\mathrm{C}_{T, K, z}^{\text {out }}(t, S)\right\}= & S \int_{\kappa \vee z}^{\kappa \vee x}(y-\kappa)\left(\psi_{\beta}(y)-\frac{\psi_{\beta}(z)}{\phi_{\beta}(z)} \phi_{\beta}(y)\right) \phi_{\beta}(x) d y \\
& +S \int_{\kappa \vee x}^{\infty}(y-\kappa)\left(\psi_{\beta}(x)-\frac{\psi_{\beta}(z)}{\phi_{\beta}(z)} \phi_{\beta}(x)\right) \phi_{\beta}(y) d y,
\end{aligned}
$$

if $x \geq z$. Note that the factor $1 / 2$ in (36) has disappeared from (37) and (38), because $w_{\beta}=1 / 2$ for squared Bessel processes.
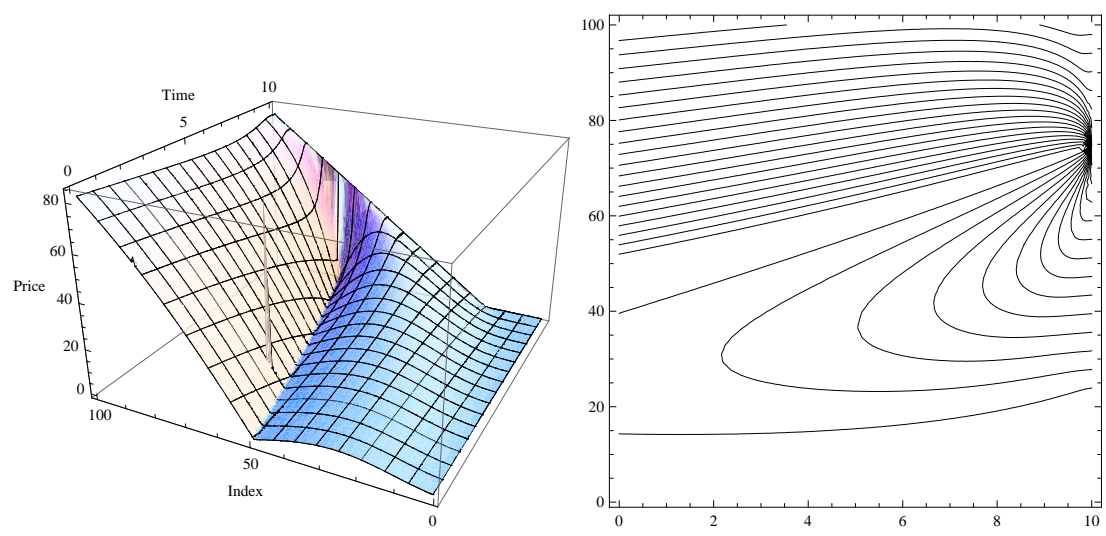

Fig. 3. The knock-out European call pricing function $\mathrm{C}_{10,20,50}^{\text {out }}(\cdot, \cdot)$

From the expressions above, we see that computing the price of a barrier option once again requires two numerical procedures: First the integrals in (37) or (38) must be evaluated by numerical quadrature, and then the Laplace transform of the option price must be inverted. Figure 3 presents the results of these procedures, in the form of surface and contour plots for the pricing function $C_{10,20,50}^{\text {out }}(\cdot, \cdot)$ of a knock-out European call with initial maturity $T=10$ years, strike $K=20$ and barrier reference level $z=50$. The values for the model parameters are the same as for the previous graphs. Firstly, we see that $C_{T, K, z}^{\text {out }}\left(t, Z_{t}\right)=0$, which agrees with the expected behaviour of the option at the knock-out barrier. Secondly, we observe that

$$
\lim _{t \rightarrow T} C_{T, K, z}^{\text {out }}(t, S)= \begin{cases}0 & \text { if } S=Z_{T} \\ (S-K)^{+} & \text {otherwise }\end{cases}
$$

in line with what we would expect close to maturity. Figure 4 makes this convergence obvious, by presenting a sequence of cross-sections of the pricing surface in Figure 3.

Acknowledgements. Thanks to Paavo Salminen for a helpful e-mail exchange on the subject of deriving transition densities for diffusions with boundary conditions. We also thank Mark Craddock for several illuminating discussions on the pitfalls of numerical Laplace transform inversion. 

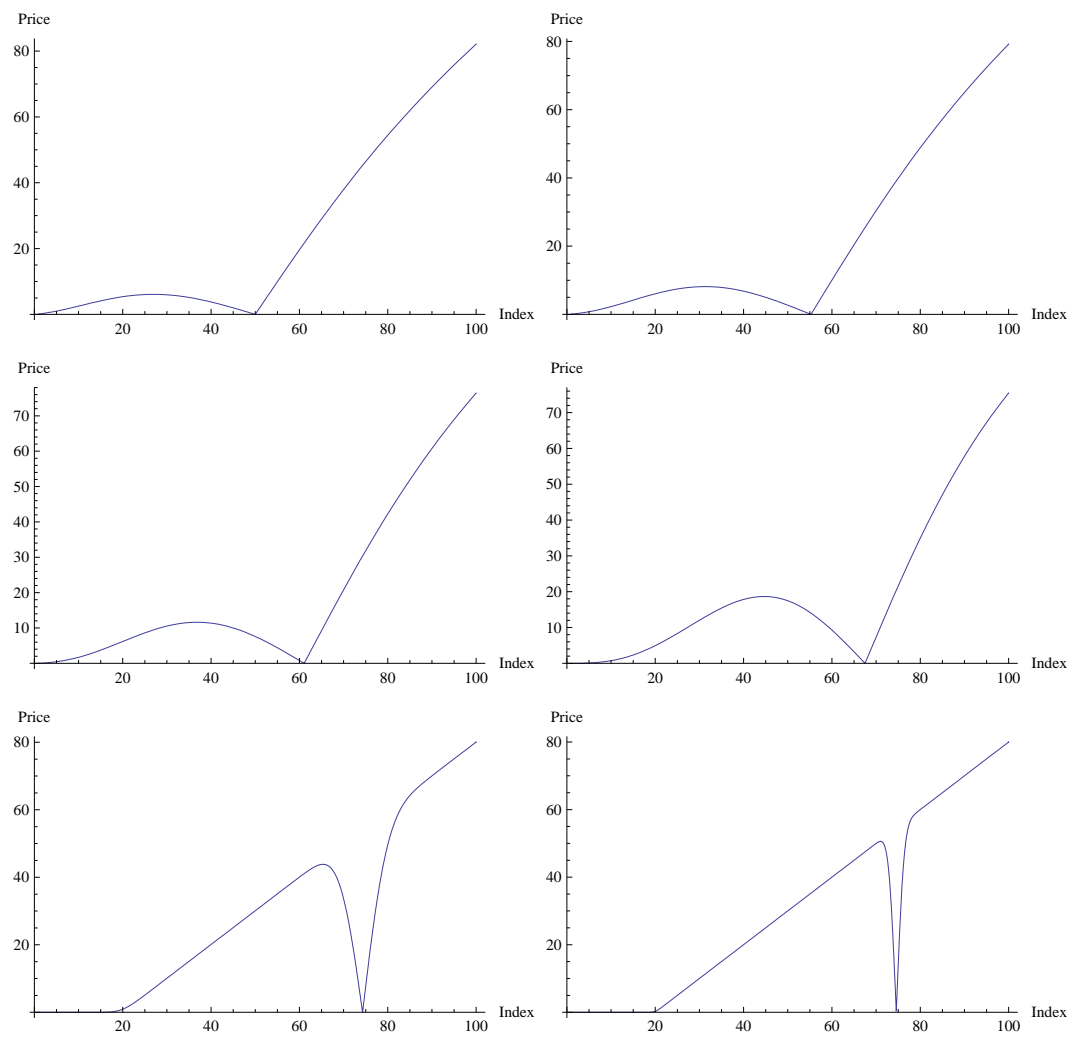

Fig. 4. Evolution of the knock-out call pricing function $\mathrm{C}_{10,20,50}^{\text {out }}(t, \cdot)$, for $t=0,2.5,5,7.5,9.9$ and 9.99 years

\section{References}

[1] J. Abate and W. Whitt, Numerical inversion of Laplace transforms of probability distributions, ORSA J. Comput. 7 (1995), 36-43.

[2] M. Abramowitz and I. A. Stegun (eds.), Handbook of Mathematical Functions, Dover, New York, 1972.

[3] F. Black, Studies in stock price volatility changes, in: Proceedings of the 1976 Business Meeting of the Business and Economic Statistics Section, American Statistical Association, 177-181, 1976.

[4] A. N. Borodin and P. Salminen, Handbook of Brownian Motion-Facts and Formulae, 2nd ed., Birkhäuser, Basel, 2002.

[5] J. C. Cox, The constant elasticity of variance option pricing model, J. Portfol. Manag. (spec. issue) (1996), 15-17.

[6] M. Craddock, D. Heath, and E. Platen, Numerical inversion of Laplace transforms: A survey of techniques with applications to derivative pricing, J. Comput. Finance 4 (2000), $57-81$.

[7] D. Davydov and V. Linetsky, Pricing and hedging path-dependent options under the CEV process, Management Sci. 47 (2001), 949-965. 
[8] D. Davidov and V. Linetsky, Structuring, pricing and hedging double-barrier step options, J. Comput. Finance 5 (2002), 55-87.

[9] F. Delbaen and H. Shirakawa, A note on option pricing for the constant elasticity of variance model, Asia-Pacific Finan. Markets 9 (2002), 85-99.

[10] M. C. Fu, D. B. Madan, and T. Wang, Pricing continuous Asian options: A comparison of Monte Carlo and Laplace transform inversion methods, J. Comput. Finance 2 (1999), 49-74.

[11] H. Geman and A. Eydeland, Domino effect, Risk 65 (1995), 65-67.

[12] H. Geman and M. Yor, Bessel processes, Asian options, and perpetuities, Math. Finance 3 (1993), 349-375.

[13] H. Geman, N. El Karoui, and J.-C. Rochet, Changes of numéraire, changes of probability measure and option pricing, J. Appl. Probab. 32 (1995), 443-458.

[14] A. Göing-Jaeschke and M. Yor, A survey and some generalizations of Bessel processes, Bernoulli 9 (2003), 313-349.

[15] D. Heath and E. Platen, Consistent pricing and hedging for a modified constant elasticity of variance model, Quant. Finance 2 (2002), 459-467.

[16] K. Itô and H. P. McKean, Jr., Diffusion Processes and their Sample Paths, Springer, Berlin, 1974.

[17] S. Karlin and H. M Taylor, A Second Course in Stochastic Processes, Academic Press, New York, 1981.

[18] J. Kelly, A new interpretation of the information rate, Bell System Tech. J. 35 (1956), 917-926.

[19] N. N. Lebedev, Special Functions and Their Applications, Dover, New York, 1972.

[20] M. Lowenstein and G. A. Willard, Local martingales, arbitrage, and viability: Free snacks and cheap thrills, Econ. Theory 16 (2000), 135-161.

[21] J. B. Long, Jr., The numeraire portfolio, J. Finan. Econ. 26 (1990), 29-69.

[22] A. Pelsser, Pricing double barrier options using Laplace transforms, Finance Stoch. 4 (2000), 95-104.

[23] G. Peskir, On the integral equations arising in the first-passage problem for Brownian motion, J. Integral Equations 14 (2002), 397-423.

[24] E. Platen and D. Heath, A Benchmark Approach to Quantitative Finance, Springer, Berlin, 2006.

[25] D. Revuz and M. Yor, Continuous Martingales and Brownian Motion, 3rd ed., Springer, Berlin, 1999.

[26] W. T. Shaw, Modelling Financial Derivatives with Mathematica: Mathematical Models and Benchmark Algorithms, Cambridge University Press, Cambridge, 1998. 
